\title{
Cases
}

\section{EUROPEAN COURT OF JUSTICE}

56/65 Société Technique Minière [1966] ECR 337

6 and 7-73, Commercial Solvents and Others v. Commission, ECR 223

27/76, United Brands Company v. Commission, ECR 207 (1978)

282

85/76, Hoffmann-La Roche \& Co. AG v. Commission, ECR 461 (1979)

$175,179,283$

258/78, Nungesser, ECR 2015 (1982)

273

311/84, Télémarketing v. CLT and IPB, ECR 3261 (1985)

177

C-241/91 P and C-242/91 P, RTE and ITP v. Commission ('Magill'), ECR I743 (1995)

177, 185, 292

C-7/97, Oscar Bronner GmbH \& Co. KG v. Mediaprint, ECR I-7791

177, 184, 185, 294

C-481/01 P(R), NDC Health v. IMS Health and Commission, ECR I-3401 (2002)

184 C-418/01, IMS Health GmbH \& Co. OHG v. NDC Health GmbH \& Co. KG, (2004)

183,293

\section{COURT OF FIRST INSTANCE}

T-51/89, Tetra Pak Rausing SA v. Commission (Tetra Pak I), ECR II-309 (1990)

126, 130, 236

T-374/94, T-375/94, T-384/94 and T-388/94, European Night Services v. Commission, ECR II-3141 (1998)

64, 67, 247

T-184/01, IMS Health v. Commission, ECR II-3193 (2001)

184

T-5/02, Tetra Laval v. Commission, ECR II-4381 (2002)

106

T-65/98, Van den Bergh Foods v. Commission (2003)

179

T-219/99, British Airways (2003)

179

\section{European Commission}

Case No IV/23.077 - Acec-Berliet, OJ L 201/7 (1968) 
Case No IV/26.917 - Henkel/Colgate, OJ L14/14 (1972) 65

Case No IV/29.151 - Video cassette recorders, OJ L 47/42 (1978) $\mathbf{1 6 2}$

Case No IV/29.479 - IBM (1984)

288

Case No IV/30.320 - Optical fibres, OJ L 236/30 (1986)

105,110 ,

$214,235,274,286$

Case No IV/30.979 and 31.394 - Decca Navigator Systems, OJ L 43/27 (1989)

295

Case No IV/31.043 - Tetra Pak I (BTG licence), OJ L 272/27

(1988)

126, 236

Case No IV/32.009 - Elopak/Metal Box-Odin, OJ L 209/15 (1990) 135, 230

Case No IV/32.363 - KSB/Goulds/Lowara/ITT, OJ L 19/25 (1991) 135, 230

Case No IV/34.776 - Pasteur Mérieux/Merck, OJ L 309/1 (1994) 150, 151,

$157,164,193,199,216,230$,

246, 259, 263, 264, 267, 274, 309

Case No IV/M.042 - Alcatel/Telettra, OJ L 122/48 (1991)

221

Case No IV/M.068 - Tetra Pak/Alfa Laval, OJ L 290/35 (1991) 61

Case No IV/M.072 - Sanofi/Sterling Drug (1991) 113

Case No IV/M.214 - Du Pont/ICI, OJ L 7/13 (1992) 98

Case No IV/M.221 - ABB/BREL, OJ C 142 (1992) 61

Case No IV/M.222 - Mannesmann/Hoesch, OJ L 114/34 (1992) 61

Case No IV/M.269 - Shell/Montecatini, OJ L 332/48 (1994) 100, 193, 202,

Case No IV/M.323 - Procordia/Erbamont, OJ C 128 (1993) 113

Case No IV/M.426 - Rhône-Poulenc/Cooper, OJ C 113 (1994) 113

Case No IV/M.457 - La Roche/Syntex, OJ C 178 (1993) 113

Case No IV/M.469 - MSG Media Service, OJ L 364/1 (1994) 271

Case No IV/M.495 - Behringwerke AG/Armour Pharmaceutical Co.,

OJ C 134/4 (1995) 113

Case No IV/M.500 - AHP/Cyanamid, OJ C 278/3 (1994) 113

Case No IV/M.555 - Glaxo/Wellcome, OJ C 65/3 (1995) 113, 140,

259, 260, 278

Case No IV/M.587 - Hoechst/Marion Merell Dow (1995) 113

Case No IV/M.603 - Crown Cork \& Seal/CarnaudMetalbox, OJ L 75/38 (1995)

97, 98, 193, 214, 227, 276

Case No IV/M.623 - Kimberly-Clark/Scott Paper, OJ L 183/1 (1996) 98

Case No IV/M.631 - Upjohn/Pharmacia, OJ C 294/9 (1995) 3, 113,

$138,147,148,211,250,272$

Case No IV/M.737 - Ciba-Geigy/Sandoz (1996) OJ L 201/1 (1997) 3, 113,

$114,121,155,157,163$,

$193,206,212,263,265,309$

Case No IV/M.877 - Boeing/McDonnell Douglas, OJ L 336/16 (1997) 228

Case No IV/M.950 - Hoffman LaRoche/Boehringer Mannheim (1997) 179 
Case No IV/M.1229 - American Home Products/Monsanto (1999) 113

Case No IV/M.1378 - Hoechst/Rhône-Poulenc (1999) 113

Case No IV/M.1397 - Sanofi/Synthélabo (1999)

113

Case No IV/M.1403 - Astra/Zeneca (1999)

113,115

Case No COMP/M.1846 - Glaxo Wellcome/SmithKline Beecham (2000) 3,

$113,114,129,148,205,207,211$, 212, 229, 230, 250, 278, 304

Case No COMP/M.1878 - Pfizer/Warner-Lambert (2000)

142,251

Case No COMP/M.2220 - General Electric/Honeywell (2001)

98

Case No COMP/M.2416 - Tetra Laval/Sidel (2001)

106

Case No COMP/M.2312 - ABBOTT/BASF (2001)

272

Case No COMP/M.2922 - Pfizer/Pharmacia (2003)

120, 129, 193,

211, 230

Case No IV/C-3/36.849 - MPEG-2 Licensing Programme, OJ C 229/19 (1998)

$157,193,260,262$

Case No IV/C-3/37.506 - DVD Patent Licensing Programme, OJ C 242/5 (1999)

$160,193,260,262$

Case COMP/C-3/37.792 - Microsoft (2004) 174, 187, 214, 288, 289, 290

Case COMP D3/38.044 - NDC Health v. IMS Health: interim measures, OJ L $59 / 18(2002)$

184

\section{US SUPREME COURT}

Hartford-Empire Co. v. United States, 323 U.S. 386 (1945) 203

Brown Shoe Co. v. United States, 370 U.S. 294 (1962) 60

United States v. Penn-Olin Chemical Co., 378 U.S. 158 (1964) 199

Walker Process Equip., Inc. v. Food Mach. \& Chem. Corp., 382 U.S. 172 (1965)

203

United States v. Grinnell Corp., 384 U.S. 563 (1966)

283

Federal Trade Commission v. Procter \& Gamble Co., 386 U.S. 568 (1967)

United States v. Falstaff Brewing Corp., 410 U.S. 526 (1973)

62

United States v. General Dynamics Corp., 415 U.S. 486 (1974)

60,61

Aspen Skiing Co. v. Aspen High-lands Skiing Corp., 472 U.S. 585 (1985)

182

Eastman Kodak Co. v. Image Technical Services, 504 U.S. 451

53,172

Spectrum Sport, Inc. v. McQuillan, 506 U.S. 447 (1993)

183

Verizon Communications, Inc. v. Law Offices of Curtis Trinko, LLP, 540 U.S. $398,(2004)$ 


\section{US CIRCUIT COURTS}

BOC International Ltd. (British Oxygen) v. FTC, 557 F.2d 24 (2d Cir. 1977)

Santa Fe-Pomeroy Inc. v. P \& Z Co., 569 F.2d 1084 (9th Cir. 1978) 234 United States v. Siemens Corp., 621 F.2d 499 (2d Cir. 1980) 60 SCM Corp. v. Xerox Corp., 645 F.2d 1195 (2d Cir. 1981), cert. denied, 455 U.S. 1016 (1982)

61, 297 MCI Communications Corp. v. AT\&T, 708 F.2d 1081 (7th Cir.), cert. denied, 464 U.S. 891 (1983)

182

Federal Trade Commission v. PPG Industries, Inc., 789 F.2d 1500 (D.C. Cir. (1986)

United States v. Microsoft Corp., 56 F.3d 1448 (D.C. Cir. 1995) 170

United States v. Microsoft Corp., 147 F.3d 935 (D.C. Cir. 1998) 170

C.R. Bard, Inc v. M3 Systems Inc. 157 F.3d 1340 (Fed. Cir. 1998) 295

Intergraph Corp. v. Intel Corp., 195 F.3d 1346 (Fed. Cir. 1999) 168

United States v. Microsoft, 253 F.3d 34 (D.C. Cir. 2001) 53, 55, 130, 170,

180, 187, 214, 224, 268, 282, 283, 285, 287, 291

\section{US District Courts}

United States v. Automobile Mfrs Ass'n., 307 F.Supp. 617 (C.D. Cal.

1969)

United States v. Black \& Decker Mfg, 430 F.Supp. 729 (D.Md. 1976) 60

Intergraph Corp. v. Intel Corp., 3 F.Supp.2d 1255 (N.D.Ala. 1998) 166, 168 FTC v. H.J. Heinz, Co., 116 F.Supp.2d 190 (D.D.C. 2000)

271

In re Papst Licensing, GmbH Patent litigation, 2000 WL 1145725 (E.D.La Aug. 11, 2000)

United States v. Microsoft Corp., 87 F.Supp.2d 30 (D.D.C. 2000) 170

United States v. Microsoft Corp., 97 F.Supp.2d 59 (D.D.C. 2000) 170 United States v. Oracle Corp., Case No. C 04-00807 VRW, (N.D. Cal. Sept. 9, 2004)

102, 219

\section{DEPARTMENT OF JUSTICE}

United States v. General Motors Corp., Civ. No. 93-530 (D. Del., filed Nov. 16, 1993)

94, 97, 98, 103, 200, 209, 214, 220

United States v. Flow International Corp and Ingersoll-Rand Co., Civ. 9471320 (E.D. Mich., filed April 14, 1994)

95, 227

United States v. Lockheed Martin Corp. and Northrop Grumman Corp (D.D.C., filed March 23, 1998) 
Xviii

United States v. Halliburton Company and Dresser Industries, Inc., Civ. No 98-CV-2340 (April 1, 1999)

96, 214, 225, 227

United States v. Compuware, Corp. and Viasoft, Inc (D.D.C., filed Oct. 29, 1999)

Varian Medical Systems (DOJ 2000)

127,237

\section{FEDERAL TRADE COMMISSION}

American Cyanamid Co., 72 F.T.C. 623 (1967) 297

Bendix Corp., Docket no. 8739 (1970)

B.A.T. Industries, Ltd., 104 F.T.C. 916 (1984) 198

Roche Holdings Ltd. 113 F.T.C. 1086 (1990) 135, 147, 148, 198,

211, 238, 248, 264

Montedison S.p.A., FTC File No. 941-0043 (Jan. 11, 1995) 98, 193, $202,214,227$

Sensormatic Elec. Corp., File No. 941-0126, 60 Fed. Reg. 5428

(Jan. 27, 1995)

131, 147, 210, 218, 307

American Home Prods. Corp., C-3557 (Feb. 14, 1995), 60 Fed. Reg. 60,807 (Nov. 29, 1994) 113, 137, 147, 149, 193, 211, 225, 239, 248, 251, 276 Boston Scientific Corp., File No. 951-0002, 60 Fed. Reg. 12,948 (Mar. 9, 1995)

111, 128, 193, 211, 229, 278

Wright Medical Technology, Inc., C-3564 (Mar. 23, 1995), 60 Fed. Reg. 460 (Jan. 4, 1995)

110, 125, 130, 193, 211, 229

Glaxo plc., FTC File No. 951-0054, 60 Fed. Reg. 16, 139 (Mar. 29, 1995)

133, 140, 149, 218, 251, 276, 278

The Upjohn Co. and Pharmacia Aktiebolag, 121 F.T.C. 44, FTC File No. 9510140, Docket no. C-3638, (Feb. 8, 1996) 139, 148, 193, 225, 249, 251, 272 Ciba-Geigy Ltd., 123 F.T.C. 842, Docket no. C-3725 (1997) 153, 163, 164, 193, 206, 215, 258, 263, 265, 276, 309

Baxter International Inc., File No. 971-0002, Docket no. C-3726 (1997)

140, 142, 148, 218, 225, 248

Digital Equipment Corporation, Docket no. C-3818 (1998)

$103,110,193$, 214, 227, 228

Summit Technology, Inc. and VISX, Inc., Docket no. C-9286 (1998) 254, 297 Intel Corporation, Docket no. 9288 (1999) $53,165,186,193$, 200, 214, 285

Hoechst AG, File No. 991-0071, Docket no. C-3919 (2000)

124, 142, 149, 193, 277

Pfizer Inc., and Warner-Lambert Company, File No. 001 0059, Docket no. C3957 (2000)

$141,142,148,211,225,230,249,251$ 
Amgen Inc. and Immunex Corporation, Docket no. C-4053 (2002) 119,

129, 193, 210, 211, 230, 277

Cytyc Corp. and Digene Corp., FTC File No. 0210098 (2002) 123, 229 Pfizer Inc. and Pharmacia Corporation, File No. 021 0192, Docket no. C4075 (2003)

120, 129, 193, 211

GenCorp Inc., Docket no. C-4099, File No. 0310152 (2003) 101, 219, 227 Genzyme/Novazyme Pharmaceuticals, Inc, File No. 021 0026, Closing letter, January 13, 2004

$123,143,144,145,147,149,193,204$, 205, 211, 213, 239, 241, 243, 248, 275, 277

MPEG Pool Letter, Business Review Letter from Joel I. Klein, Acting Assistant Attorney General, Antitrust Division, Department of Justice, to Garrard R. Beeney, Esq. (June 26, 1997)

157, 160, 260, 262

DVD Pool Letter. Business Review Letter from Joel I. Klein, Acting Assistant Attorney General, Antitrust Division, Department of Justice, to Garrard R. Beeney, Esq. (Dec. 16, 1998)

$160,260,262$

3G Patent Platform Partnership. Business Review Letter from Charles A. James, Assistant Attorney General, Antitrust Division, Department of Justice, to Ky P. Ewing, Esq. (November 12, 2002)

161 
Marcus Glader - 9781847201683

Downloaded from PubFactory at $\odot 4 / 26 / 2023$ 09:0०:54AM via free access 